\title{
Study on Operational Availability Relational Model of RMS
}

\author{
Kai Cao ${ }^{1,2}$, Zheng Huang ${ }^{1,2}$ and Xiaowei Liu ${ }^{1,2}$ \\ ${ }^{1}$ College of Power Engineering, Naval University of Engineering, Wuhan, China \\ ${ }^{2}$ Military Key Laboratory for Naval Ship Power Engineering, Naval University of Engineering, Wuhan, China \\ Email:kevin10001@163.com
}

\begin{abstract}
This paper is about a research of the relationship between equipment's reliability, maintainability, supportability and operational availability. It investigates the influencing factors of operational availability, analyzes the maintenance strategies of equipment and specifies distribution types. Based on these findings and Markow process, it then deduces computational model of operational availability. It also defines reliability, maintainability, supportability and other constraint condition and establishes optimizing equations to find feasible solution and optimal solution. At last, it tests correctness and feasibility of established model by case study. After knowing the requirement of operational availability and operational method provided here to define corresponding level of reliability, maintainability, supportability, it transforms operational-using requirement to technical design requirement in support for further research.
\end{abstract}

\section{Introduction}

Operational availability is one of the vital parameters in the development and application of warship equipment. The warship equipment needs to guarantee various missions carried out away from shore base, which requires equipment readiness to fully complete tasks assigned. Paying attention to the design of operational availability, as the key connotation in optimizing the operational effectiveness of warship equipment, will aid the concept transformation from solely stress on the performance to pursuing comprehensive mission effectiveness [1]. Operational availability is coguaranteed by the design reliability, maintainability and supportability of the system; hence the process of designing the operational availability is also the process of confirming the reliability, maintainability and supportability of the equipment, converting operational requirements to technical design requirements.

Numerous researches had been taken on the evaluation of operational availability home and abroad, of which the common methods are classical model method, statistic analyzing method and analytical method.Sherbrooke divides the operational availability model into attainability availability and supply availability according to its definition, which is widely applied in the inventory field [2]. Diao et al established the calculation model based on the steady state availability with full consideration of the operational availability of three states of unmanned aircraft system - stored, combat and sealed [3]. Based on the statistical analysis of failure data, Liu et al raised a combined GM-RBF neural network model on grey theory [4]. Liu and Han et al discussed the application of BP neural network and RBF neural network on the evaluation of operational availability [5,6]. Zhang et al descry ibed a statistical evaluation method tightly related to the missile weapon system by analyzing the availability of different equipment using statistical method and connecting the system structure based on in situ data [7].Zhang et al studied the lower confidence limit of operational availability of single element product on a given distribution [8]. De Smidt-Destombes K S and Zhang et al established calculation model of the $\mathrm{k} / \mathrm{N}$ warm standby reductant system's operational availability when the overhaul interval is set based on the Markov process [9,10]. Chan G K and Liu deduced a relation model among steady state availability and distribution, delayed maintenance time distribution, maintenance time distribution and preventative maintenance time distribution $[11,12] . \mathrm{Xu}$ and Duan et al established the naval gun operational availability model $[13,14]$.

It is known that great deal of results have been accumulated on the operational availability by related researchers. The classical model method is easy to calculate and suits to solve the inventory, however the results might be quite different from the simulation ones [15]. The statistic analyzing method requires relatively long period to gather data, moreover it cannot be applied to the equipment development stage [11]. In addition, the researches focused more on the already known maintenance strategy, system structure, and distribution types, and thus deduce the calculation models of the operational availability, but the researches on the other way around was quite few. 
Due to the questions above, this article will investigate the factors influencing the operational availability, analyze the maintenance strategy, and determine the distribution types, and deduce the calculation model of operational availability based on Markov process; determine the reliability, maintainability, supportability and other limiting factors, establish the optimized equation set and its feasible solutions and optimal solution. Verify the validity and feasibility of the established model through case study.

\section{Models of operational availability}

The warship equipment needs to guarantee various missions carried out away from shore base, and the resources taken on the ship is limited while the support resources is hard to replenish, in order to guarantee the equipment readiness, a deep study on the operational availability is what it calls for to establish the models to calculate operational availability, and offer theoretical support to improve designation and optimize allocation of resources.

Before the models are established, some explanations and assumptions are made as follows:

(1) Two levels of spare parts supply system are considered, assume that all spare parts in local level can be fulfilled in the intermediate level warehouse.

(2) Assume that local level spare parts have 3 individuals, and when the third ran out, a request for the 4th is made to the base. If the equipment faces no absence of any failure before the spare parts' arrival, 3 parts will be supplied, and the extra ones will be sent back to the base; if the equipment faces any failure and caused delay, the failed one will be changed and 3 more supplied. The time from application to acquirement is $t$, which obeys index distribution with parameter.

(3) The longevity and maintenance time obeys index distribution with and parameter, and can be analyzed through Markov process.

(4) When the spare parts are used up and the requested part doesn't arrive but the equipment fails, thus is the logistice delay until the part arrives, mean logistic delay time is the average time of logistic delay.

(5) When any failure is noticed of the equipment, immediate maintenance should be made to its normal form. Only replacement of the equipment is made on the ship and the maintenance staff is enough at hand.

The working and local level storage conditions are expressed with $E_{m n}$. The subscript $\mathrm{m}$ stands for the working condition of the equipment, 0 for normal working condition, 1 for logistic delay condition, 2 for maintenance condition. The subscript $\mathrm{n}$ stands for local warehouse condition, 0 for the spare parts ran out and request to intermediate level warehouse for supply is already made, 1 for 1 spare part, 2 for 2 spare parts, 3 for 3 spare parts, therefore 9 conditions are defined, $E_{03}$ stands for normal working condition with 3 spare parts; $E_{22}$ stands for under maintenance with 2 spare parts; $E_{02}$ stands for normal working condition with 2 spare parts; $E_{21}$ stands for under maintenance with 1 spare part; $E_{01}$ stands for normal working condition with 1 spare part; $E_{20}$ stands for under maintenance with 0 spare part; $E_{00}$ stands for normal condition with 0 spare part; $E_{10}$ stands for under maintenance with 0 spare part; $E_{23}$ stands for under maintenance with 3 spare parts. We consider any condition that doesn't satisfy the assumptions doesn't exist.

The time of requesting for the spare part obeys index distribution with $\gamma$ parameter, the final spare part's life span also obeys index distribution with $\lambda$, parameter, therefore the supplied spare part can arrive before the last available element fails(event A) or after(event B). These two events should be discussed separately.

According to the assumption, when the last spare part is used, the application for more spare part is made, that is the last spare part come into working condition and the application is made coincide. If random variable $\mathrm{X}$ is used to express the elements' lifespan, $Y$ to express the application time, then the possibility of event A happens is $P_{A}(X \geq Z)$, possibility of event $\mathrm{B}$ happens is $P_{B}(X \leq Z)$. Because the time of applying for spare part obeys index distribution with $\gamma$ parameter, the longevity of the element obeys also index distribution with $\lambda$ parameter, plus $\mathrm{X}$ and $\mathrm{Y}$ are independent, so the combined probability density function are as follows:

$$
f(x, z)=\lambda \gamma e^{-\gamma z} e^{-\lambda x}
$$

The solution of $P_{B}(X \leq Z)$

$$
\begin{aligned}
& P_{B}(X \leq Z)=\iint_{D_{x z}} f(x, z) d x d z=\frac{\lambda}{\lambda+\gamma} \\
& P_{A}(X \geq Z)=1-P_{B}(X \leq Z)=\frac{\gamma}{\lambda+\gamma}
\end{aligned}
$$

$\mathrm{D}_{\mathrm{xz}}$ means the range where $\mathrm{X}$ is less than or equal to $\mathrm{Z}$.

Assume that failure happens at $t_{1}$, and the spare part arrives at $t_{2}$, then:

$$
\begin{aligned}
& F\left(t_{1}\right)=1-e^{-\lambda t 1}, G\left(t_{2}\right)=1-e^{-\gamma t_{2}} \\
& f\left(t_{1}\right)=\lambda e^{-\lambda t 1}, g\left(t_{2}\right)=\gamma e^{-\gamma t_{2}}
\end{aligned}
$$


Due to the fact that the failures happens and the spare part arrives are two independent events, so the combined probability density function is :

$$
h\left(t_{1}, t_{2}\right)=\lambda \gamma e^{-\gamma t 2} e^{-\lambda t 1}
$$

If the spare part arrives after the last element fails, it will cause delay for $\left(t_{2}-t_{1}\right)$. Regardless of the delay time caused by other factors, the mean logistic delay time is:

$$
M L D T=E\left(t_{2}-t_{1}\right)
$$

According to the two-dimensional random variable function and its distribution, we can conclude that:

$$
E\left(t_{2}-t_{1}\right)=\iint_{D t 1 / 2}\left(t_{2}-t_{1}\right) h\left(t_{1}, t_{2}\right) d t_{1} d t_{2}=\frac{\lambda}{\gamma(\lambda+\gamma)}
$$

Among them $\mathrm{D}_{\mathrm{t} 1 \mathrm{t} 2}$ represents a range $t_{1} \leq t_{2}$.

In the following, we will take consideration of event A. Complementary element arrived before the last available element had broken. At this moment, the condition aggregate $\mathrm{E}_{\mathrm{A}}$ is $\{1,2,3,4,5,6,7\}$, normal condition aggregate $\mathrm{W}_{\mathrm{A}}$ is $\{1,3,5,7\}$, and breakdown condition aggregate $F_{A}$ is $\{2,4,6\}$. The figure of condition transformation is reflected in Figure 1 .

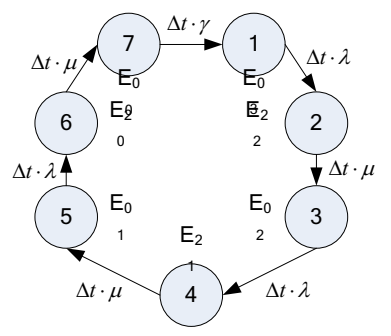

Fig. 1. Condition transformation of event A.

Condition transformation matrix $\mathrm{Q}_{\mathrm{A}}$ can be showed in the following from condition transformation figure:

$$
\mathbf{Q}_{\mathrm{A}}=\left(\begin{array}{lllllll}
-\lambda & \lambda & & & & & \\
& -\mu & \mu & & & & \\
& & -\lambda & \lambda & & & \\
& & & -\mu & \mu & & \\
& & & & -\lambda & \lambda & \\
& & & & & -\mu & \mu \\
\gamma & & & & & & -\gamma
\end{array}\right)
$$

According to bibliography [16] :

$$
\left(P_{1}^{\prime}(t), P_{2}^{\prime}(t), \cdots, P_{7}^{\prime}(t)\right)=\left(P_{1}(t), P_{2}(t), \cdots, P_{7}(t)\right) \mathbf{Q}_{\mathrm{A}}
$$

As for all $\mathrm{i}, \mathrm{j} \in \mathrm{E}, \lim _{t \rightarrow \infty} P_{i j}(t)=P_{j} \quad j=1,2, \cdots, 7$.

As for any $\mathrm{j} \in \mathrm{E}, \lim _{t \rightarrow \infty} P_{j}^{\prime}(t)=0$

We can choose term limit value from both sides according to equation (10), and then obtain:

$$
\left(P_{1}, P_{2}, \cdots, P_{7}\right) \mathbf{Q}_{\mathrm{A}}=(0,0, \cdots, 0)
$$

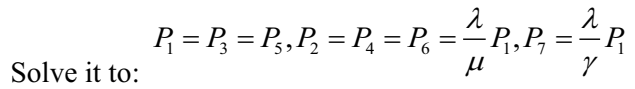

Due to $P_{1}+P_{2}+\cdots P_{7}=1$, we can make a conclusion:

$$
P_{1}=\left(3+\frac{3 \lambda}{\mu}+\frac{\lambda}{\gamma}\right)^{-1}
$$


According to the definition of $A_{\mathrm{o}}$, we know:

$$
\begin{aligned}
& A_{o \mathrm{~A}}=P_{1}+P_{3}+P_{5}+P_{7} \\
& =\left(3+\frac{\lambda}{\gamma}\right)\left(3+\frac{3 \lambda}{\mu}+\frac{\lambda}{\gamma}\right)^{-1}
\end{aligned}
$$

When we take consideration of event B, complementary element arrived after the last available element had broken, and maintenance delay condition occurred before re-complementary element's arrival. At this moment, the condition aggregate $E_{B}$ is $\{1,2,3,4,5,6,7,8,9\}$, normal condition aggregate $W_{B}$ is $\{1,3,5,7\}$, and breakdown condition aggregate $F_{B}$ is $\{2,4,6,8,9\}$. The figure of condition transformation is reflected in Figure 2.

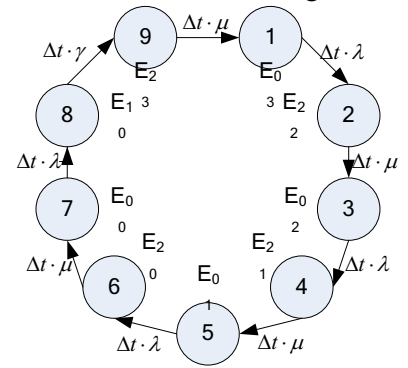

Fig. 2. Condition transformation of event.

Condition transformation matrix QBcan be showed in the following from condition transformation figure:

According to bibliography 16 :

$$
\mathbf{Q}_{\mathrm{B}}=\left(\begin{array}{llllllllll}
-\lambda & \lambda & & & & & & & \\
& -\mu & \mu & & & & & & \\
& & -\lambda & \lambda & & & & & \\
& & & -\mu & \mu & & & & \\
& & & & -\lambda & \lambda & & & \\
& & & & & -\mu & \mu & & \\
& & & & & & -\lambda & \lambda & \\
& & & & & & & -\gamma & \gamma \\
\mu & & & & & & & & & \\
& & & & & & & & -\mu
\end{array}\right)
$$

$$
\left(P_{1}^{\prime}(t), P_{2}^{\prime}(t), \cdots, P_{y}^{\prime}(t)\right)=\left(P_{1}(t), P_{2}(t), \cdots, P_{9}(t)\right) \mathbf{Q}_{\mathrm{B}}
$$

As for all $\mathrm{i}, \mathrm{j} \in \mathrm{E}, \lim _{t \rightarrow \infty} P_{i j}(t)=P_{j} \quad j=1,2, \cdots, 9$

As for any $\mathrm{j} \in \mathrm{E}, \lim _{t \rightarrow \infty} P_{j}^{\prime}(t)=0$.

We can choose term limit value from both sides according to equation (15), and then obtain:

$$
\left(P_{1}, P_{2}, \cdots, P_{9}\right) \mathbf{Q}_{\mathrm{B}}=(0,0, \cdots, 0)
$$

Solve it to:

$$
\begin{aligned}
& P_{1}=P_{3}=P_{5}=P_{7}, P_{8}=\frac{\lambda}{\gamma} P_{1}, \\
& P_{2}=P_{4}=P_{6}=P_{9}=\frac{\lambda}{\mu} P_{1} 。
\end{aligned}
$$

Due to $P_{1}+P_{2}+\cdots P_{9}=1$, we can make a conclusion:

$$
P_{1}=\left(4+\frac{4 \lambda}{\mu}+\frac{\lambda}{\gamma}\right)^{-1}
$$

According to the definition of $A_{o}$, we know : 


$$
\begin{aligned}
& A_{o \mathrm{~B}}=P_{1}+P_{3}+P_{5}+P_{7} \\
& =4 \cdot\left(4+\frac{4 \lambda}{\mu}+\frac{\lambda}{\gamma}\right)^{-1}
\end{aligned}
$$

Combining $\mathrm{P}_{\mathrm{A}}$ and $\mathrm{P}_{\mathrm{B}}$, we can make a conclusion of systematic comprehensive $A_{o}$ :

$$
\begin{aligned}
A_{o} & =P_{A} \cdot A_{o \mathrm{~A}}+P_{B} \cdot A_{o \mathrm{~B}} \\
& =\frac{\gamma}{\lambda+\gamma} \cdot\left(3+\frac{\lambda}{\gamma}\right)\left(3+\frac{3 \lambda}{\mu}+\frac{\lambda}{\gamma}\right)^{-1}+\frac{4 \lambda}{\lambda+\gamma} \cdot\left(4+\frac{4 \lambda}{\mu}+\frac{\lambda}{\gamma}\right)^{-1} \\
& =\frac{3 \gamma+\lambda}{\lambda+\gamma}\left(3+\frac{3 \lambda}{\mu}+\frac{\lambda}{\gamma}\right)^{-1}+\frac{4 \lambda}{\lambda+\gamma} \cdot\left(4+\frac{4 \lambda}{\mu}+\frac{\lambda}{\gamma}\right)^{-1}
\end{aligned}
$$

Ao , MTBF,MTTR and MLDT can be calculated according to known parameter $\lambda, \mu$ and $\gamma$ in model (8)and (19). But thisset of parameters may not be the optimal parameters. In the process of equipment designation, we concern more about limit value of $A_{o}$ and the feasible solution and optimal solution of design parameter limited by other term, thus we need to come up with an optimized method.

\section{Ascertain limit term and establish optimal relational models}

According to $A_{o}$, the larger $M T B F$ is, the smaller MTTR is, the smaller MLDT is and the higher $A_{o}$ is. But in fact, as the result of the limitation of technical level and other objective conditions, MTBF, MTTR and MLDT can only change in a range, so do parameter $\lambda, \mu$ and $\gamma$. Besides, maintenance delay time (event B) made by the drawn-out application of replacement part can be cut down by optimized allocation of maintenance system, so we can prescribe a limit that maintenance delay time takes up proportion in unusable time no more than limit value. Unusable time is made of average maintenance delay time and average repair time. Maintenance delay time taking up proportion in unusable time no more than limit value is equal to average repair time taking up proportion in unusable time more than limit value. Then regard available cost's limit value, range of $\lambda, \mu$ and $\gamma$, the rate average repair time takes up in unusable time, average maintenance delay time as limit condition, and the maximal value of $A_{o}$ as target function, to establish optimized relational models:

$$
\left\{\begin{array}{l}
\max A_{\mathrm{o}}(\lambda, \mu, \gamma)= \\
\frac{3 \gamma+\lambda}{\lambda+\gamma}\left(3+\frac{3 \lambda}{\mu}+\frac{\lambda}{\gamma}\right)^{-1}+\frac{4 \lambda}{\lambda+\gamma} \cdot\left(4+\frac{4 \lambda}{\mu}+\frac{\lambda}{\gamma}\right)^{-1} \\
\text { s.t. } A_{\mathrm{o}} \geq A_{\text {oaccept }} \\
a \leq E\left(t_{1}\right)=\frac{1}{\gamma} \leq b \\
\quad \leq M T B F=\frac{1}{\lambda} \leq d \\
e \leq M T T R=\frac{1}{\mu} \leq f \\
g \leq M L D T=\frac{\lambda}{\gamma(\lambda+\gamma)} \leq h \\
\quad M T R R \\
\quad M T T R+M L D T
\end{array}\right.
$$

We can choose reciprocal from both sides of equation according to $A_{o}$ equation, and then obtain 17:

$$
M T T R+M L D T=\left(\frac{1}{A_{o}}-1\right) M T B F
$$

so we can present the rate average repair time takes up in unusable time in the following:

$$
\frac{M T T R}{M T T R+M L D T}=\frac{M T T R}{\left(\frac{1}{A_{o}}-1\right) M T B F}
$$

\section{Solve the equation set and analyze the case}


There are three variables $\lambda, \mu$ and $\gamma$ in optimal relational model (20), which both has linear constraint and nonlinear constraint, belonging to multivariate nonlinear constraint optimized problem. According to optimal theory, we can obtain optimal solution which meets the limit condition and optimal value of corresponding parameters by solving optimal relational model through numerical value MATLAB 18.

We know that under the limitation of current technical level and other usage conditions, some equipment's $M T B F$ is more than $500 \mathrm{~h}$ and less than $1000 \mathrm{~h}$, MTTR is larger than $4 \mathrm{~h}$ and less than $25 \mathrm{~h}$, and average time from the application of replacement parts to the arrival of them is more than $72 \mathrm{~h}$ and less than $120 \mathrm{~h}$, as well as $A_{\text {oaccept }}$ is 0.96 , lower limit value of $M L D T$ is $g$, and the rate MTTR takes up in unusable time should be more than $k$,so the optimal relational model (20) can be transformed into (23).

$$
\left\{\begin{array}{l}
\max A_{\mathrm{o}}(\lambda, \mu, \gamma)= \\
\frac{3 \gamma+\lambda}{\lambda+\gamma}\left(3+\frac{3 \lambda}{\mu}+\frac{\lambda}{\gamma}\right)^{-1}+\frac{4 \lambda}{\lambda+\gamma} \cdot\left(4+\frac{4 \lambda}{\mu}+\frac{\lambda}{\gamma}\right)^{-1} \\
\text { s.t. } A_{\mathrm{o}} \geq 0.96 \\
72 \leq E(t)=\frac{1}{\gamma} \leq 120 \\
500 \leq M T B F=\frac{1}{\lambda} \leq 1000 \\
4 \leq M T T R=\frac{1}{\mu} \leq 25 \\
g \leq M L D T=\frac{\lambda}{\gamma(\lambda+\gamma)} \\
\frac{M T T R}{\left(\frac{1}{A_{o}}-1\right) M T B F} \geq k
\end{array}\right.
$$

In the relational model, $\lambda, \mu$ and $\gamma$ are variables. After performing the procedure, we can obtain the maximal value and corresponding parameters of $A_{0}$. Then change the rate $k$ which MTTR takes up in unusable time and lower limit value $g$ of $M L D T$, and re-perform the procedure to obtain the maximal value and corresponding parameters of different $A_{0}$. Data are showed in the following table:

Table 1. The maximal value of $A_{\mathrm{o}}$ and corresponding parameters.

\begin{tabular}{|c|c|c|c|c|c|c|c|}
\hline No. & $\boldsymbol{k}$ & $\boldsymbol{g} / \mathbf{h}$ & $\boldsymbol{m a x} \boldsymbol{A}_{\boldsymbol{o}}$ & $\boldsymbol{M T B F} / \mathbf{h}$ & $\boldsymbol{M T T R} / \mathbf{h}$ & $\boldsymbol{E}(\boldsymbol{t}) / \mathbf{h}$ & $\boldsymbol{M L D T} / \mathbf{h}$ \\
\hline 1 & 0.8 & 5 & 0.9943 & 1000 & 4.6 & 73.4 & 5.02 \\
\hline 2 & 0.85 & 5 & 0.9926 & 1000 & 6.4 & 73.4 & 5.02 \\
\hline 3 & 0.8 & 6 & 0.9932 & 1000 & 5.5 & 81.1 & 6.08 \\
\hline 4 & 0.85 & 6 & 0.9912 & 1000 & 7.6 & 81.1 & 6.08 \\
\hline 5 & 0.8 & 7 & 0.9921 & 1000 & 6.4 & 87.4 & 7.03 \\
\hline 6 & 0.85 & 7 & 0.9901 & 1000 & 8.5 & 87.4 & 7.03 \\
\hline
\end{tabular}

When $\mathrm{k}$ is 0.85 and $\mathrm{g}$ is $6 \mathrm{~h}$, we can obtain relation figure showed in Figure 3 according to the relation between expected time $E(t)$ from replacement parts' application to their arrival and $\mathrm{A}_{0}$.

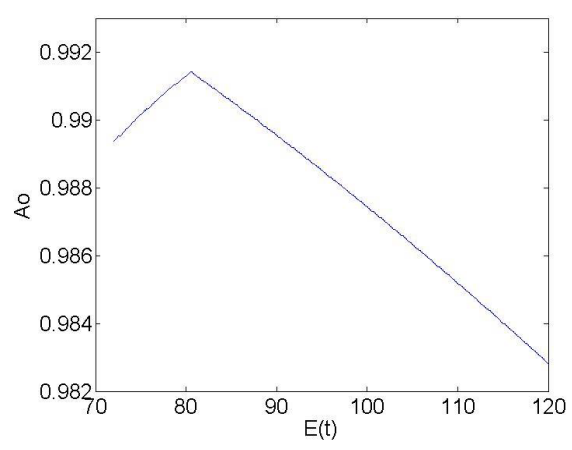

Fig. 3. The relation between $E(t)$ and $A_{0}$. 
After calculating, when $E(t)$ is $81.1 \mathrm{~h}, \mathrm{~A}_{\mathrm{o}}$ arrives at the maximal value. At this moment, the three dimensional relational model of MTTR, MTBF and Ao can be showed in Figure 4.

From Table 1, we know that when $\mathrm{k}$ are same, the smaller $\mathrm{g}$ is, the larger the maximal value of operational availability is, and MTTR, MLDTand $E(t)$ will gradually decrease. When $g$ are same, the smaller kis, the larger the maximal value of operational availability is, and the optimal value of other parameters won't change, but only the optimal value of MTTR will decrease. The optimal value of MTBFwill choose the maximal value of the range all the time. Combining the relationship figure of $M T B F, M T T R$ and $\mathrm{A}_{o}$, as well as relationship figure of $E(t)$ and $A_{o}$, we will analyze the phenomenon above.

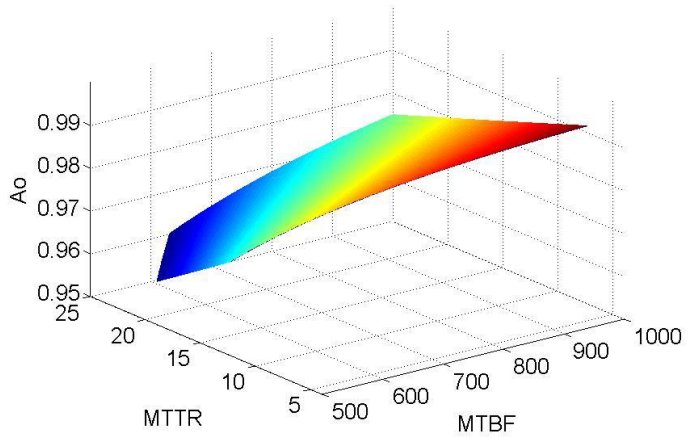

Fig. 4. The relation between $M T T R, M T B F$ and $A_{o}$.

According to formula (8), we know that $M L D T$ is influenced by $\lambda$ and $\gamma$, it is inversely proportional to MTBF, and it is in proportion to $E(t)$. When lower limit $\mathrm{k}$ that average repair time takes up proportion in unusable time are same, the lower limit value gof $M L D T$ decreases and the constraint that MLDTputs on MTBF and $E(t)$ also decreases. At this moment, $M T B F$ will be bigger in its co-domain and $E(t)$ will be smaller in its co-domain, as reflected in Figure 4 that the maximal value of $\mathrm{A}_{\mathrm{o}}$ then will increase.

According to formula(22), we know that $\mathrm{k}$ is in proportion to MTTR and inversely proportional to MLDT. When $\mathrm{g}$ are same, and $\mathrm{k}$ decreases, the constraint that the lower limit that average repair time takes up proportion in unusable time puts on MTTR and MLDTalso decreases. At this moment, MTTR will be smaller in its co-domain, as reflected in Figure 4 that the maximal value of $\mathrm{A}_{o}$ then will increase. Although the constraint that $\mathrm{k}$ puts on $M L D T$ decreases, the constraint that $\mathrm{g}$ puts on $M L D T$ doesn't change, so that the optimal value of other parameters won't change.

We can know from Figure(4) that when $\mathrm{k}$ is $0.85, \mathrm{~g}$ is $6 \mathrm{~h}$, and $\mathrm{t}$ is $81.1 \mathrm{~h}, \max _{\mathrm{o}}$ that has concerns constraint conditions is the maximal value 0.9912 as $M T B F$ is $1000 \mathrm{~h}$. When $M L D T$ is inversely proportional to $M T B F$, and it is in proportion to $E(t), M L D T$ 's contour line can be showed in Figure(5). Under current constraint conditions, when $g$ is between $5 \mathrm{~h}$ and $7 \mathrm{~h}$, the minimal value of $M L D T$ constrains the range of $M T B F$ 's and $E(t)$ 's feasible region to be pentagon area in the lower right corner above contour line gall the time. When $\mathrm{A}_{0}$ is the maximal value, MTBFcan always be the maximal value $1000 \mathrm{~h}$ in its feasible region. If taking costs, time limit and other constraints into consideration, $M T B F$ 's value should be further restricted.

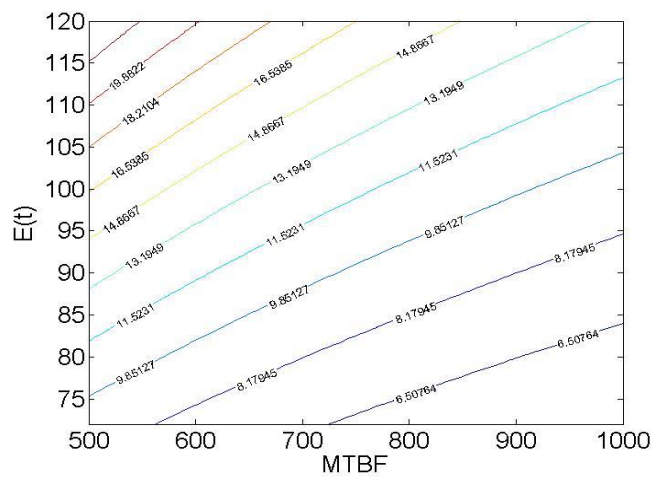

Fig. 5. Contour line of $M L D T$.

\section{Conclusion}

Operational availability is an integrity norm as same importance as other functional norms of equipment, and it is an insurance of equipment that it can finish tasks perfectly when needed. This paper analyzes the influencing factors of 
operational availability and has a research on the process of replacement parts' maintenance based on fully understanding of the importance of operational availability. And based on Markow process, it establishes relational models of operational availability and defines constraint condition and establishes optimizing equations to find feasible solution and optimal solution. At last, it tests correctness and feasibility of established model by case study. The arithmetic established here transforms operational-using requirement to technical design requirement and ascertains target of equipment's reliability, maintainability and supportability. It provides theoretical support and quantitative reference of parameter setting when testing equipment.

Although the arithmetic provided here can solve some physical problem, the optimal solution may have some distinction to reality without consideration of costs, limit time and other constraints. So it is the main problem we should solve in the future.

\section{References}

1. HJB 31.2-03, Management guidance of operational availability of naval ships' equipment.

2. Sherbrooke C C.Optimal inventory modeling of system: multi-echniques second edition. 371(2004)

3. W.Q. Diao, S.W. Lu, B. Zheng. Analysis of unmanned combat aircraft storage security and operational availability.Aircraft design , 35, 48(2015)

4. W. Liu, T.K. Li, S.C. Yu. Study on appraisal method of missile weapon system's operational availability based on neural network of GM-RBF. Equipment environmental engineering, 10, 108(2013)

5. Q.J. Liu, G.M. Chen, X.F. Liu, etc. An application of BP neural network in the prediction of missile weapon system's operational availability. Tactical missile technology,1, 26(2011)

6. Z. Han, T.X. Xu, J.K. Yang. Study on neural network of RBF in the prediction of missile weapon system's operational availability. Computers and modernization, 10, 27(2012)

7. H.X. Zhang, C.H. Wang. Study on appraisal method of ground-to-air missile weapon system's operational availability. Modern defense technology, 43,129(2015)

8. G.F. Zhang, H.P. Wu. Assessment of steady state availability of repairable system when maintenance delay time occurs. Journal of Zhejiang University, 33, 0268(2006)

9. De Smidt-Destombes K S, van der Heijden M C, van Harten A. Availability of k-out-of-N systems under block replacement sharing limited spares and repair capacity. International Journal of Production Economics, 107, 404(2007)

10. T. Zhang, J.J. Zhang, Guo B. Analysis of maintenance strategies of K/N system based on operational availability. Acta Astronautica, 30, 395(2009)

11. F.S. Liu, W. Wu, Z.W. Shan, etc. Operational availability model of armored equipment based on Markov updating process. Journal of armored corps engineering college, 24,15(2010)

12. Chan G K, Asgarpoor S. Optimum Maintenance Policy with Markov Processes. Electric Power Systems Research, 76,452(2006)

13. T.G. Xu, Y. Wei.Operational availability modeling and simulation of shipborne gun based on spare parts inventory. Equipment environmental engineering, 7,132(2010)

14. M.Q. Duan, J. Dai, J. Ma. Operational availability simulation of task oriented marine nuclear power plant. Traffic science and technology, 2,160(2015)

15. Y. Wang, N.C Wang, L. Ma, ec.Computational method of multi-unit tadem system's operational availability concerned constraint conditions of spare parts. Aeronautical Journal, 36,1195(2015)

16. J.H. Cao, K. Chen.An introduction to mathematics of reliability.(2005)

17. Y.G Zhao, C Qi, Y.L. Shang. Optimization of equipment's operational availability under the condition of two level protection. Science and technology of atomic energy, 47,1202(2013)

18. C. Gong, Z.L Wang. Frequently-used algorithm assembly of MATLAB language.(2008) 